events obligatorily linked to the usage. Smoking can be considered a learned (conditioned) behavior reinforced by nicotine. Those events associated with nicotine are also subject to conditioning. For smoking, the drug-taking behavior is associated with common events of the day, such as waking in the morning or the evening meal. After repeated conditioning, these drug-associated cues themselves motivate smoking. A recent functional magnetic resonance imaging study of addicted smokers showed that cues related to smoking elicit neural activity in regions linked to attention, memory, emotion and motivation ${ }^{13}$. In rats, presentation of nicotine-associated cues reinstated previously extinguished nicotineseeking behavior, providing evidence that links nicotine-associated cues to relapse ${ }^{14}$.

The drug-related associations that motivate use can also be aversive. For example, environmental cues linked to the acute symptoms of withdrawal may stimulate ongoing drug use. Withdrawal from chronic nicotine administration decreases 'reward' signaling, as detected by decreases in evoked dopamine release and elevated thresholds for intracranial self stimulation in rats ${ }^{10,15}$. Stimuli repeatedly paired with withdrawal discomfort come to elevate reward thresholds on their own. Thus, the changes in reward pathways normally caused by nicotine withdrawal eventually arise from conditioned stimuli that then cue drug use to relieve the symptoms ${ }^{10}$. The conditioned, neutral stimuli gain incentive salience that prompts continued nicotine use. Also, in human addicts, exposure to environmental situations previously paired with withdrawal provides a powerful cue for drug use. The withdrawal cue induces an aversive internal state that prompts normally adaptive behaviors to relieve that state. In this case, unfortunately, the behavior is continued drug use.

In summary, drug addiction subverts normal mechanisms of neuronal adaptation, learning and memory; leading to long-lasting changes in behavior that accrue with the ongoing progression of addiction ${ }^{9,11}$. Normal behavior develops as a function of experiences, such as those associated with adaptive reward-based learning, which is the essential process subverted by addiction ${ }^{11}$. Events and processes associated with the addictive drug (for example, the environment during drugtaking) become linked to the acquisition and delivery of drug, making them salient cues that prompt the desire for the drug 9 .

Patients with lesions to the insula often quit smoking easily and lose the subsequent desire to smoke $^{4}$, and the insula is active during the urge for drugs ${ }^{7,8}$. Thus, a lesion to the insula seems to disrupt the representation of internal states ${ }^{5,6}$ that underlie the motivation or urge for the drug (nicotine in this case). The accumulating evidence (albeit indirect) indicates that learned drug associates can cue internal states that perpetuate addiction. After the insula is lesioned, learned associations to the drug experience no longer produce the internal bodily states that drive drug use, particularly during periods of abstinence.

\section{COMPETING INTERESTS STATEMENT}

The authors declare no competing financial interests.

1. WHO. World Health Organization, http://www.who.int/ tobacco/helath_priority/en/print.html (2007).

2. Balfour, D.J. Nicotine Tob. Res. 6, 899-912 (2004).

3. Dani, J.A. \& Harris, R.A. Nat. Neurosci. 8, 1465-1470 (2005).

4. Naqvi, N.H., Rudrauf, D., Damasio, H. \& Bechara, A. Science 315, 531-534 (2007).

5. Craig, A.D. Nat. Rev. Neurosci. 3, 655-666 (2002).

6. Damasio, A.R. et al. Nat. Neurosci. 3, 1049-1056 (2000).

7. Bonson, K.R. et al. Neuropsychopharmacology 26 376-386 (2002).

8. Kilts, C.D. et al. Arch. Gen. Psychiatry 58, 334-341 (2001).

9. Everitt, B.J., Dickinson, A. \& Robbins, T.W. Brain Res. Brain Res. Rev. 36, 129-138 (2001).

10. Kenny, P.J. \& Markou, A. J. Neurosci. 25, 6208-6212 (2005).

11. Hyman, S.E., Malenka, R.C. \& Nestler, E.J. Annu. Rev. Neurosci. 29, 565-598 (2006).

12. Schultz, W., Dayan, P. \& Montague, P.R. Science 275, 1593-1599 (1997).

13. Smolka, M.N. et al. Psychopharmacology (Berl.) 184 577-588 (2005).

14. Paterson, N.E., Froestl, W. \& Markou, A. Neuropsychopharmacology 30, 119-128 (2005).

15. Volkow, N.D., Fowler, J.S., Wang, G.J. \& Goldstein, R.Z. Neurobiol. Learn. Mem. 78, 610-624 (2002).

\title{
Developing recognition of faces and places
}

In adults, high-level visual cortex includes several regions that seem to be specialized for processing different classes of stimuli, such as faces or objects. However, little is known about the developmental trajectory of these areas or how this progression relates to aptitude in object, face or scene recognition. A paper on page 512 by Golijeh Golarai and colleagues provides an elegant demonstration of the concurrent changes in these specialized cortical regions and recognition memory during development. Their findings suggest that cortical maturation varies temporally across functional regions and is correlated with the development of category-specific recognition memory.

The authors used fMRI to identify areas of the occipito-temporal cortex that preferentially respond to objects (lateral occipital complex, LOC), faces (fusiform face area, FFA) or places (parahippocampal place area, PPA) in children, adolescents and adults. They found that children had smaller face-sensitive and place-sensitive cortical areas than adults, but no age-related

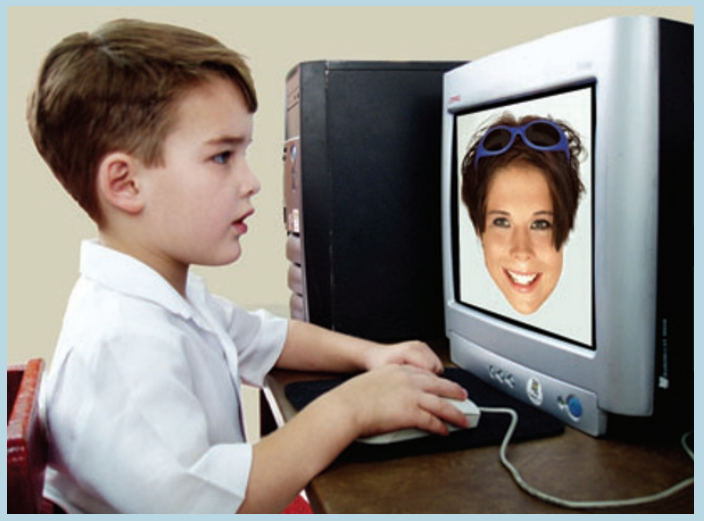
changes in the size of object-selective cortical areas. In these same subjects, tests of recognition memory for faces, objects and places revealed that age was correlated with face and place recognition memory, whereas there were no effects of age on memory for objects. Finally, the authors went a step further to show that face recognition memory correlated with the size of the right FFA, and that place recognition memory correlated with the size of the left PPA. Object recognition memory was not correlated with the size of any of the cortical areas of interest.

These changes were specifically associated with improvements in recognition memory for faces and places, but not for objects, suggesting a more prolonged development of the FFA and PPA than the LOC, lasting at least until children reached the age of 11 . One possible implication of this finding is that there may be an important role for experience even in the development of high-level visual cortex. In addition, the finding that better recognition memory was associated with increases in the size of specific cortical areas provides hints about the potential coding schemes that could be used in these areas to represent complex stimuli.

Hannah Bayer 